\title{
0 uso da tecnologia da informação e comunicação para caracterizar os municípios: quem são e o que precisam para desenvolver ações de governo eletrônico e smart city
}

\author{
Erico Przeybilovicz ${ }^{1}$ \\ Maria Alexandra Cunha' \\ Fernando de Souza Meirelles ${ }^{1}$ \\ 1 Fundação Getulio Vargas / Escola de Administração de Empresas de São Paulo, São Paulo / SP — Brasil
}

\begin{abstract}
Este estudo explora as características de infraestrutura e uso das tecnologias da informação e comunicação dos municípios brasileiros, refletindo sobre a possibilidade de desenvolverem ações de governo eletrônico e smart cities. Um estudo quantitativo de análise de agrupamentos foi realizado sobre dados da Munic 2014 e identificou quatro clusters: o Sem-tecnologia, o Atento ao cidadão, o Atento à legislação e o Provido de TIC, que sugerem diferentes vias de ação em TIC. Cada grupo de municípios apresenta diferentes necessidades para o desenvolvimento de ações de governo eletrônico e smart cities, que requerem ações específicas para cada um dos quatro clusters.
\end{abstract}

Palavras-chave: smart city; município; TIC; governo eletrônico.

El uso de la tecnología de la información y comunicación para caracterizar a los municipios: quienes son y lo que necesitan para desarrollar acciones de gobierno electrónico y smart city

Este estudio investiga las características de infraestructura y uso de las tecnologías de la información y comunicación de los municipios brasileños, reflexionando sobre la posibilidad de desarrollo de acciones de gobierno electrónico y de smart cities. Un estudio cuantitativo de análisis de agrupamientos realizado sobre datos de la investigación Munic-2014 identificó cuatro clusters: el Sin-tecnología, el Atento al Ciudadano, el Atento a la legislación y el Provisto de TIC, que sugieren diferentes vías de acción en TIC. Cada grupo de municipios presenta diferentes necesidades para el desarrollo de acciones de gobierno electrónico y smart cities que indican la necesidad de acciones específicas para cada uno de los cuatro clusters.

Palabras clave: smart city; municipio; TIC; gobierno electrónico.

\section{The use of information and communication technology to characterize municipalities: who they are and what they need to develop e-government and smart city initiatives}

This study investigates the infrastructure characteristics and use of information and communication technologies of Brazilian municipalities reflecting on the possibility of developing e-government and smart cities actions. A quantitative study of cluster analysis, using data from the research "Munic 2014", identified four clusters: the Technologyless, the Concerned-on citizen, the Concerned-on legislation, and the ICT Supported, that suggest different courses of action in ICT. Each group of municipalities presents different needs for the development of e-government and smart cities actions that indicate the need for specific actions for each of the four clusters.

Keywords: smart city; municipality; ICT; e-government. 


\section{INTRODUÇÃO}

Este trabalho tem por objetivo compreender as características de infraestrutura e uso de TIC dos municípios para desenvolverem ações de governo eletrônico (eGov) e iniciativas de smart cities. Ao longo dos anos, os países vêm avançando nos indicadores de governo eletrônico (United Nations, 2016) e cidades de todas as regiões do planeta estão implementando ações para se tornarem mais inteligentes, criando soluções urbanas fortemente baseadas no emprego de tecnologia. Quer-se melhorar a vida das pessoas que habitam as cidades, ampliar a qualidade da gestão dos recursos e contar com a participação dos cidadãos.

Embora o tema de smart city seja estudado por áreas como planejamento urbano e gestão de tecnologia em governo, entre outros campos de conhecimento, até o momento parece não haver um consenso sobre a definição do termo (Bolívar, 2016). Utiliza-se o termo smart associado ao desenvolvimento de soluções para problemas urbanos baseadas em tecnologia. O tema eGov é mais antigo e, talvez por isso, mais difundido e estudado. Está vinculado à modernização da administração pública por meio do uso de tecnologia da informação e comunicação (TIC), à melhoria da eficiência dos processos operacionais e administrativos dos governos (Diniz et al., 2009), e também pode ser associado ao uso de internet no setor público para prestação de serviços online e para a ampliação da prática democrática (Cunha e Miranda, 2013). Tanto as iniciativas de eGov como as de smart cities dependem de infraestrutura tecnológica, do conjunto de hardware, software, redes, internet, serviços e aplicações, e do uso que os governos locais fazem desse acervo. No Brasil, os projetos de governo eletrônico vêm sendo implantados desde a década de 2000. Mais recentemente, há várias iniciativas de smart cities: a Frente Nacional de Prefeitos abriga a Rede de Cidades Inteligentes e Humanas; o Programa Minha Cidade Inteligente do Ministério da Ciência, Tecnologia, Inovações e Comunicações; está em formalização, no Congresso Nacional, a Frente Parlamentar Mista em apoio às cidades inteligentes e humanas; Curitiba, Vitória e Rio de Janeiro são alguns exemplos de cidades com iniciativas smart; agências multilaterais e bancos de desenvolvimento estão implementando linhas de financiamento para essa área e, por óbvio, fornecedores de tecnologia, em escala mundial, estão fortemente interessados no tema. Nas eleições municipais de outubro de 2016, smart city e governo eletrônico foram pauta dos planos de governo de vários candidatos. No entanto, e apesar dos esforços, a gestão de TIC nas cidades brasileiras é deficitária, o contexto brasileiro é de baixa agregação de tecnologia aos processos governamentais, infraestrutura deficiente de tecnologia da informação e comunicação, e grandes desafios a superar na governança e gestão de TIC (CGI, 2016a).

A premissa que guia este trabalho é que a disponibilidade de infraestrutura nos municípios é diversa, com consequências para o uso que as prefeituras fazem dos recursos tecnológicos. Essas diferenças de disponibilidade e uso de tecnologia configuram diferentes desafios e caminhos a seguir, quando da implantação de projetos de eGov ou de smart city. Assim, a pergunta de pesquisa que orienta o presente trabalho é: como a infraestrutura e o uso de TIC caracterizam grupos de municípios? Ao identificar esses grupos, a elaboração de recomendações de políticas de gestão que os considerem potencializa os ganhos de investimentos em tecnologia. Para a construção da resposta foram utilizadas técnicas estatísticas descritivas e de agrupamentos, com base em dados disponibilizados pelo Instituto Brasileiro de Geografia e Estatística (IBGE) na Pesquisa de Informações Básicas dos Municípios Munic 2014 (IBGE, 2016) - sobre uso e disponibilidade de infraestrutura de TIC.

Este trabalho traz contribuições para a prática e para o campo de estudos sobre o uso de TIC em governos. Em relação à prática governamental, gestores públicos e especialistas em agências mul- 
tilaterais, que conduzem e projetam programas de fomento ao uso de TIC, podem se beneficiar do entendimento das diferenças entre municípios. Os programas e linhas de apoio e/ou financiamento devem considerar essas diferenças no planejamento de investimentos em infraestrutura. Do ponto de vista científico, Gregor (2006) descreve os trabalhos em sistemas de informação que elaboram taxonomias ou tipologias como teoria para "analisar". A tipologia de municípios oferecida neste trabalho revela características da informatização de governos locais no Brasil e permite que estudos subsequentes aprofundem o entendimento do fenômeno, com a criação de indicadores, testes ou ampliação da explicação sobre a prática brasileira.

\section{GOVERNO ELETRÔNICO, SMART CITIES E A INFRAESTRUTURA DE TIC NOS MUNICÍPIOS BRASILEIROS}

A literatura de eGov e smart cities confere especial relevância à infraestrutura de TIC em governos locais. Os primeiros estudos no campo, europeus e americanos, foram seguidos por pesquisas nos países em desenvolvimento. A partir da conceituação de eGov e smart cities, esta seção mostra a infraestrutura de TIC como condição necessária, ainda que não suficiente, para a materialização dos ganhos potenciais do eGov. O Brasil já tem dados robustos que permitem refletir sobre este tema.

Governo eletrônico, ou eGov, pode ser entendido como o uso de TIC pelos governos no relacionamento com os cidadãos e no desenvolvimento da administração pública (Grönlund, 2010). Esse termo surgiu no final dos anos 1990, muito associado a movimentos de reforma do Estado e à nova gestão pública. No início, o termo estava mais ligado à prestação de serviços eletrônicos e obtenção de eficiência administrativa, mas, mais recentemente, e até em publicações não acadêmicas, incorporou ao seu escopo o suporte dos meios digitais à prática democrática (United Nations, 2016:143). Inúmeros trabalhos dedicaram-se a conceituar eGov e mapear a área sob diferentes perspectivas, tais como a disponibilização de e-serviços ao cidadão (Rampelotto, Löbler e Visenti, 2015; Damian e Merlo, 2013), o uso de TIC para os processos internos da administração pública (Tripadalli, Fernandes e Machado, 2011), transparência, governança e democracia (Pinho, 2008; Bernardes, Santos e Rover, 2015) e estágios de desenvolvimento de eGov no Brasil (Sano, 2012). Há recomendação de que se façam estudos sobre os fatores que promovem ou dificultam a adoção de modernas ferramentas de TIC pelos municípios (Sano, 2012). No Brasil, há amplas investigações sobre o uso de TIC pelos governos. O IBGE conduz as pesquisas Estadic e Munic, com censo de dados nos estados e municípios. O Comitê Gestor da Internet no Brasil publica a TIC Domicílios (CGI, 2016b), que contempla o uso de eGov pela população, e TIC Governo Eletrônico (CGI, 2016a), que aprofunda aspectos de infraestrutura de TIC, uso e oferta de serviços e mecanismos de participação.

O desenvolvimento de infraestrutura de TIC tem um efeito positivo no desenvolvimento e crescimento de eGov, principalmente a internet. As ações de eGov fazem uso da infraestrutura e do acervo de informações governamentais para fornecer transparência e e-serviços. Quando a infraestrutura de TIC é deficiente, o desenvolvimento do eGov é inibido e limita a proporção de cidadãos que podem ser atendidos (Singh, Das e Joseph, 2007; Siau e Long, 2009; Srivastava e Teo, 2010; Zhao, Collier e Deng, 2014). Os governos têm considerado as TIC como uma ferramenta importante para atingir metas, como a melhoria da qualidade dos serviços públicos, menores custos e aumento da transparência. Os países em desenvolvimento também veem as TIC como ferramentas indispensáveis, mas não suficientes, para o desenvolvimento econômico e social e redução da corrupção. O exemplo da experiência do México deixa claro que a introdução das TIC é um componente de reforma do governo, 
mas outras mudanças se fazem necessárias, tanto no aspecto organizacional do governo como em questões legais e culturais (Gil-Garcia, Arellano-Gault e Luna-Reyes, 2010). A infraestrutura de TIC é especialmente relevante para o avanço de eGov em governos locais. Os governos locais são o nível mais próximo do cidadão, entre as instituições do Estado. Os municípios, condados, distritos são responsáveis pela prestação de serviços públicos locais, de acordo com as leis de cada país (Rahman, 2010). O estudo de eGov em governos locais teve início nos Estados Unidos e na Europa (Almazan e Castañeda, 2010), mas já há estudos sobre outras regiões do planeta, em especial nos países em desenvolvimento. Nos Estados Unidos, Kaylor, Deshazo e Van Eck (2002) analisaram os municípios e o uso de TIC para o desenvolvimento de serviços públicos na web, propondo meios para aferir a implementação de eGov entre diferentes cidades americanas. Leenes e Svensson (2002) identificaram diferenças no desenvolvimento de eGov, entre o nível nacional e municipal na Holanda, e verificaram que, enquanto o governo nacional apresentava sucesso, os municípios eram lentos e ineficientes, com poucos recursos para implantação de eGov. Moon (2002) identificou resultados semelhantes na adoção de eGov nos governos locais estadunidenses, e sugeriu que há barreiras para o progresso do eGov municipal como a falta de capacidade financeira, técnica, de pessoal e questões legais. Na África observou-se que a falta de infraestrutura de TIC restringe seriamente o uso da internet e a adoção do eGov, não apenas em governos locais, mas em todo o continente africano (Ngulube, 2007). A partir das ações de eGov na Nigéria, sugere-se fortalecer a infraestrutura de TIC para melhorar a oferta de e-serviços e poder empreender novos projetos (Asogwa, 2013). Uma revisão comparativa sobre estudos das estratégias de eGov, realizados em governos locais de seis países (Bangladesh, Índia, Correia do Sul, Paquistão, Singapura e Sri Lanka), levou a concluir que a ampliação de eGov depende do desenvolvimento institucional, da criação de infraestrutura de informação, adequação legal e regulatória, e que o governo central tem um papel importante para subsidiar sua implantação (Rahman, 2010).

Os estudos parecem concordar que, para alcançar as capacidades máximas que as TIC podem oferecer à prática de eGov, é necessário levar em consideração o contexto legal e socioeconômico, as demandas pragmáticas locais, incluir os participantes no processo de gestão local e reforçar as capacidades institucionais (Lalovic, Djukanović e Zivkovic, 2004). Ter um plano acordado em nível nacional, a integração entre os departamentos do governo e iniciativas planejadas para implementar políticas de infraestrutura de TIC são importantes, assim como as parcerias, tanto locais como internacionais, para financiar o progresso das iniciativas de eGov em países em desenvolvimento (Thompson e Brown, 2007). O investimento em TIC pode não levar ao aumento da utilização e difusão de eGov, necessitando educação e formação comportamental dos cidadãos (Zhao, Collier e Deng, 2014). No entanto, o desenvolvimento da infraestrutura de TIC, bem como a oferta intensiva de e-serviços pelo governo local, poderá engajar grupos de cidadãos ativos nos processos de tomada de decisão e apoiar o desenvolvimento e implementação adequada de e-democracia na localidade (Turner, 2005).

O termo smart city é mais recente do que eGov. Está ligado a políticas e ideais de planejamento originários dos Estados Unidos, em particular, ao conceito de crescimento inteligente desenvolvido no âmbito do novo urbanismo, na década de 1980, e que mais tarde alcançou a Europa (Hollands, 2008). O adjetivo smart envolve principalmente a relação entre espaço urbano e tecnologia, e inclui questões como a capacidade de gerar inovação, a transição para formas de governança eletrônica, aprendizagem social, e a possibilidade de fornecer infraestruturas de TIC (Castells e Hall, 1994; Hollands, 2008). Smart city deriva da sobreposição e combinação dessas duas vertentes, o novo ur- 
banismo e a onda de desenvolvimento das TIC (Hollands, 2008; McFarlane, 2011). Embora o termo smart city seja utilizado entre os profissionais de tecnologia da informação e também em círculos acadêmicos, uma compreensão clara e consistente do conceito ainda não foi alcançada (Chourabi et al., 2012; Bolívar, 2016). Existe uma gama de definições que parecem centrar-se em três diferentes tipos de definição ideal: smart cities como as cidades que utilizam tecnologias inteligentes (foco tecnológico), smart cities como cidades com pessoas inteligentes (foco em pessoas) e smart cities como cidades com colaboração inteligente (foco na governança) (Meijer e Bolívar, 2016). Em geral, nas definições, os governos são chamados a desempenhar um papel fundamental na promoção e no desenvolvimento das smart cities, utilizando as TIC na criação interativa, participativa e baseada na informação coletada dos ambientes urbanos (Batagan, 2011; Batty et al., 2012). A necessidade de TIC para ser smarter está na forma como os governos atuam e não só na maneira como as cidades inteligentes geram capital e riqueza (Hollands, 2008). Eles podem utilizar a capacidade das tecnologias baseadas na internet para entender a sociedade mais plenamente, melhorar a participação da cidadania em processos de tomada de decisão, aumentar a eficiência dos serviços públicos e melhorar a transparência e accountability (Bolívar, 2016).

Os projetos de smart cities caracterizam-se então como esforços conscientes para usar as TIC com o objetivo de transformar a vida e o trabalho em uma região, de forma significativa e fundamental, em vez de incremental (Komninos e Sefertzi, 2009). Tal como em eGov, a TIC não é o único ingrediente dos projetos, mas sem tecnologia não é possível construir uma smart city (Malek, 2009; Cunha et al., 2016). Para Nam e Pardo (2011), existem três componentes fundamentais de uma cidade inteligente: tecnologia (incluindo infraestrutura de hardware e de software), pessoas e instituições. A infraestrutura de TIC (hardware, software, redes, internet, dados etc.) da cidade é importante, pois, por um lado, o cidadão precisa ter o acesso à tecnologia para usufruir das inovações e serviços disponibilizados. Por outro, as empresas e o governo necessitam de tecnologia para oferecer serviços. Assim, uma parte dos estudos sobre as ações de smart cities aborda questões de infraestrutura tecnológica e tecnologias facilitadoras, tentando entender as tensões entre acessibilidade tecnológica e a infraestrutura e disponibilidade de sistemas (Giffinger e Gudrun, 2010). Mudanças tecnológicas ocorrem de forma rápida e são um desafio para o planejamento dos governos locais e para o desenvolvimento das comunidades urbanas (Anttiroiko, 2012), uma vez que é necessário levar em conta o potencial de integração entre as infraestruturas de TIC e seus processos operacionais (Comissão Europeia, 2012), bem como a possibilidade de participação dos cidadãos na governança da cidade por meios eletrônicos.

A compreensão do campo brasileiro de eGov e de smart cities passa pelo entendimento do uso de TIC nos municípios. No Brasil, o acesso à internet já atende 51\% dos domicílios (CGI, 2016b), o equivalente a 34,1 milhões de residências. Considerando apenas os domicílios urbanos, esse número aumenta para 56\%, enquanto nas áreas rurais é de 22\%. Segundo estimativa da Fundação Getulio Vargas (Meirelles, 2016), o Brasil possui quatro dispositivos (computadores, tablets ou telefones inteligentes) para cada cinco habitantes. Os indicadores de eGov mostram que, nas prefeituras, o acesso a computadores e à internet está universalizado (CGI, 2016a). Mas ter acesso universal a recursos não torna os municípios iguais no uso e aplicação de TIC. Há desafios de infraestrutura de comunicação, de sistemas, no provimento de serviços ao cidadão, em transparência e em mecanismos de participação (CGI, 2016a), e há diferentes condições institucionais e capacidades técnicas (Przeybilovicz, Silva e Cunha, 2015). Em relação à gestão dos recursos de TI, nem metade das prefeituras (41\%) possui uma área ou departamento de TI e 23\% das prefeituras que dizem ter área de TI não 
têm qualquer funcionário efetivo, são contratos provisórios (CGI, 2016a). Se todos os municípios possuem computadores e internet, levantam-se as questões sobre quem está efetuando a compra e a gestão desses recursos, quem é responsável pela tomada de decisão de arquitetura, de sistemas, de relacionamento com os fornecedores. A pesquisa TIC Governo Eletrônico (CGI, 2016a) desenha um cenário de frágeis estruturas de TIC nos municípios e elas podem ter papel fundamental para garantir os interesses e demandas dos cidadãos em relação ao acesso à internet, por exemplo (Gonçalves, 2011). Outras pesquisas corroboram a modesta preocupação das cidades com as TIC, embora quase todas tenham fixado em seus planos diretores a proposta de construção de sistemas de informação (Jambeiro, Sobreira e Rabeiro, 2011).

A literatura percorrida oferece indícios de que a infraestrutura de TIC é condição básica para o desenvolvimento de ações de eGov e de smart cities. Neste estudo, buscam-se compreender as características de TIC dos municípios, pois diferentes configurações de infraestrutura sugerem diferentes caminhos de ação.

\section{PROCEDIMENTOS METODOLÓGICOS}

Este é um trabalho quantitativo que utiliza técnicas descritivas e de agrupamentos. Com o auxílio do software SPSS ${ }^{\circledast}$, foram utilizadas estatísticas descritivas para organização, sumarização e descrição do conjunto de dados. Em seguida, os dados foram submetidos a técnicas multivariadas interdependentes de análise de agrupamento, que permitem o reconhecimento de padrões. Segundo essa técnica, os agrupamentos resultantes devem então exibir elevada homogeneidade interna (dentro dos agrupamentos) e elevada heterogeneidade externa (entre agrupamentos) (Hair et al., 1998). No trabalho, isso significa dizer que os municípios incluídos em um grupo devem ter características semelhantes de infraestrutura e de uso de TI, e que cada grupo de municípios é heterogêneo em relação aos demais grupos.

A população é formada pelos 5.570 municípios brasileiros. Os dados foram coletados a partir da base de dados da Munic, realizada pelo IBGE, em 2014. A Munic tem edição periódica, sendo a primeira realizada em 1999 e a última em 2015 em todos os municípios, configurando um censo. A publicação da Munic é organizada em capítulos, nos quais são destacados aspectos relevantes da gestão e da estrutura dos municípios. Neste artigo foram utilizadas as respostas dos municípios sobre comunicação e informática da Munic 2014, uma vez que a pesquisa de 2015 não coletou dados sobre esses temas. Esse bloco possui 85 questões sobre as formas de atendimento a distância, informática, comunicação, inclusão digital e acesso à informação pública. Também foram consultados os sites do Programa das Nações Unidas para o Desenvolvimento (PNUD), que traz o Atlas do Desenvolvimento Humano (IDH-M); a pesquisa Pibmun 2012, que fornece estimativas do Produto Interno Bruto per capita por município, e a base de dados do Sistema de Informações Contábeis e Fiscais do Setor Público Brasileiro (Siconfi), da Secretaria do Tesouro Nacional, que disponibiliza informações do balanço anual de 2014, declarados pelos representantes legais dos municípios.

As variáveis de tecnologia foram reagrupadas em quatro dimensões: infraestrutura de conexão da prefeitura, inclusão digital, e-serviços e comunicação com o público, e e-transparência, conforme proposto por Przeybilovicz, Silva e Cunha (2015) e fundamentado na proposta de Cunha e Miranda (2013) (figura 1). Como o estudo utiliza uma base de dados secundária, nem todas as dimensões propostas podem ser completamente descritas. A figura 1 mostra, em retângulos, as dimensões que 
são mais comuns aos aspectos de governança ou eGov e os que foram observados. A aplicação das TIC pode ser feita nas áreas de políticas de gestão ou de políticas públicas, e-serviços públicos e ampliação da prática democrática. Neste estudo, os dados secundários da Munic permitiram observar aspectos de e-serviços que preveem a melhoria na prestação de serviços ao cidadão, por meios digitais, e de transparência, possibilitada pelo uso das TIC. Como outros aspectos têm sido valorizados nos projetos de uso de TIC pelos governos, os círculos da figura 1 representam essas outras dimensões. Foi possível examinar dados a respeito de governança de TIC (infraestrutura de conexão) e inclusão digital. A inclusão digital compreende os esforços governamentais para o uso de internet, acesso aos meios de comunicação, a um computador, e mesmo às habilidades de sua utilização pelos cidadãos.

\section{FIGURA 1 MODELO REFERENCIAL DE USO DE TIC PELA ADMINISTRAÇÃO PÚBLICA NO BRASIL}



Fonte: Adaptada de Cunha e Miranda (2013).

A partir da inspeção visual e análise descritiva dos dados extraídos da Munic, foram excluídos os municípios de Brejo de Areia (MA) e Hulha Negra (RS), pois não responderam a nenhuma das questões. Foram também excluídos os 733 municípios que não responderam a nenhuma pergunta sobre e-serviços e comunicação. A amostra final é composta por 4.835 municípios. Em termos de variáveis, foram excluídas as que não estavam relacionadas com o uso de TIC, as nomeadas como "outros", as qualitativas e as variáveis sobre possuir computadores em funcionamento (100\%) e internet $(99,8 \%)$. Ao final, foram utilizadas 39 variáveis, entre as 85 disponibilizadas pela Munic. Constatou-se que 
municípios importantes no contexto nacional ficavam fora da amostra por não terem respondido a algumas das 39 questões. Esses municípios tiveram seus dados completados pelos pesquisadores e validados por três especialistas na área. Foram incluídas na base as variáveis IDH-M (PNUD), PIB per capita (IBGE) e receita per capita do município (STN). Para esta última variável, naqueles que não apresentavam informação, considerou-se a receita média de municípios com o mesmo porte populacional, segundo a classificação do IBGE.

O modelo final é composto por 4.835 municípios, com as 39 variáveis apresentadas no quadro 1, que compõem as quatro dimensões analisadas; $\mathrm{e}$ as informações socioeconômicas dos municípios utilizadas para caracterização. Todas as variáveis de tecnologia possuem respostas dicotômicas 0 (não) e 1 ( $\operatorname{sim}$ ), com exceção da variável computadores ligados em rede, 0 (não), 1 (parte dos computadores) e 2 (todos os computadores).

\section{QUADRO 1 DIMENSÕES E VARIÁVEIS DE ANÁLISE}

\begin{tabular}{|c|c|c|}
\hline Dimensões & Variáveis & Breve descrição \\
\hline $\begin{array}{l}\text { Infraestrutura de } \\
\text { conexão da Prefeitura }\end{array}$ & $\begin{array}{l}\text { Computadores ligados em rede } \\
\text { Existência de intranet } \\
\text { Página ativa na internet do governo municipal }\end{array}$ & $\begin{array}{l}\text { As variáveis representam os recursos } \\
\text { de infraestrutura de conexão e TIC }\end{array}$ \\
\hline $\begin{array}{l}\text { e-Serviços e } \\
\text { Comunicação com o } \\
\text { cidadão }\end{array}$ & $\begin{array}{l}\text { Disponibiliza formas de atendimento a distância } \\
\text { Comunicação pela internet } \\
\text { Disponibiliza serviços informativos do município e notícias } \\
\text { Acesso a documentos e formulários } \\
\text { Licitações } \\
\text { Ouvidoria serviço de atendimento ao cidadão } \\
\text { Pregão eletrônico } \\
\text { Consulta a processos } \\
\text { Consulta prévia obtenção de alvará provisório } \\
\text { Diário oficial, legislação municipal e finanças públicas } \\
\text { Concursos públicos } \\
\text { Matrícula escolar na rede pública online } \\
\text { Emissão de certidão negativa de débito } \\
\text { Agendamento de consulta na rede pública de saúde }\end{array}$ & $\begin{array}{l}\text { As variáveis contemplam as } \\
\text { formas de atendimento a distância/ } \\
\text { eletrônico e os serviços públicos } \\
\text { disponibilizados à população de } \\
\text { forma online na internet }\end{array}$ \\
\hline
\end{tabular}




\begin{tabular}{|c|c|c|}
\hline Dimensões & Variáveis & Breve descrição \\
\hline Inclusão digital & $\begin{array}{l}\text { Governo desenvolve política/plano/programa de inclusão digital } \\
\text { Disponibiliza acesso público gratuito à internet em centros } \\
\text { de acesso } \\
\text { Infs. de serviços disponibilizados em quiosque/balcão } \\
\text { informatizado } \\
\text { Estabelecer ponte entre parceiros e comunidades carentes } \\
\text { (aquisição de TIC) } \\
\text { Criação de telecentros } \\
\text { Instalação de computadores na rede pública de ensino } \\
\text { Governo garante acesso e conexão via WiFi }\end{array}$ & $\begin{array}{l}\text { As variáveis ilustram as ações de } \\
\text { inclusão digital desenvolvidas pelo } \\
\text { governo local }\end{array}$ \\
\hline e-Transparência & $\begin{array}{l}\text { Existe legislação específica/procedimentos/providências LAl } \\
\text { Disponibiliza meios para solicitação/acesso a informação pública } \\
\text { Solicitações recebidas governo municipal (2013) } \\
\text { Disponibiliza em tempo real informações da execução } \\
\text { orçamentária e financeira } \\
\text { Registro das competências } \\
\text { Estrutura organizacional } \\
\text { Endereços e telefones das unidades organizacionais } \\
\text { Horários de atendimento ao público } \\
\text { Registros, repasses ou transferências de recursos } \\
\text { financeiros } \\
\text { Registros das despesas } \\
\text { Informações concernentes a procedimentos licitatórios } \\
\text { Dados gerais para o acompanhamento de programas } \\
\text { Respostas às perguntas mais frequentes da sociedade } \\
\text { Publicados em formato aberto } \\
\text { Há atualização de dados }\end{array}$ & $\begin{array}{l}\text { As variáveis apresentam as ações } \\
\text { do governo local para disponibilizar } \\
\text { acesso a informações públicas } \\
\text { utilizando meios eletrônicos }\end{array}$ \\
\hline $\begin{array}{l}\text { Informações } \\
\text { socioeconômicas }\end{array}$ & $\begin{array}{l}\text { População do município (2014) } \\
\text { PIB per capita do município (2012) } \\
\text { IDH-M (2010) } \\
\text { Receitas correntes per capita (2014) }\end{array}$ & $\begin{array}{l}\text { As variáveis ilustram as condições } \\
\text { socioeconômicas dos municípios }\end{array}$ \\
\hline
\end{tabular}

Fonte: Elaborado pelos autores. 


\section{ANÁLISE DOS DADOS E APRESENTAÇÃO DOS RESULTADOS}

$\mathrm{Na}$ análise descritiva percebe-se que serviços mais avançados são pouco disponibilizados na internet, 90,5\% dos municípios não disponibilizam consulta/obtenção de alvará, 98,3\% não possuem matrícula escolar online e $98,9 \%$ não possuem agendamento médico online. Os sites das prefeituras têm características predominantemente informativas (91,6\%). Os municípios desenvolvem ações de inclusão digital (87,3\%) como o acesso público à internet $(59,4 \%)$, criação de telecentros $(51,9 \%)$ e instalação de computadores na rede pública de ensino (66,2\%). Sobre a transparência, 78,5\% ainda não possuem legislação específica para atendimento à Lei de Acesso à Informação (LAI).

A análise de agrupamentos foi realizada com as variáveis de tecnologia apresentadas no quadro 1. Para a geração dos clusters foram experimentados diferentes métodos no SPSS ${ }^{\circledast}$ v.21.0. Num primeiro passo, realizou-se a análise de "duas etapas" e obteve-se a indicação de quatro grupos. Num segundo passo, realizou-se a análise com método Ward e distância euclidiana. O dendograma indicou a existência de três clusters. Por fim, utilizou-se o K-means. Estudos anteriores (Przeybilovicz, Cunha e Quandt, 2014; Przeybilovicz, Silva e Cunha, 2015) sugeriam a formação de seis clusters. Foram realizadas análises para três, quatro, cinco e seis clusters. Escolheu-se a análise de quatro clusters porque os agrupamentos resultantes apresentaram maior possibilidade de explicação pelos pesquisadores. Acrescentaram-se as médias das variáveis socioeconômicas para melhor descrição (tabela 1).

\section{TABELA 1 RESULTADO DA ANÁLISE DE CLUSTERS K-MEANS}

\begin{tabular}{|c|c|c|c|c|c|c|}
\hline \multicolumn{7}{|c|}{ Centro final dos clusters } \\
\hline \multirow{2}{*}{ Dimensões } & \multirow{2}{*}{ Variáveis } & \multirow{2}{*}{ Range } & \multicolumn{4}{|c|}{ Cluster } \\
\hline & & & 1 & 2 & 3 & 4 \\
\hline \multirow{3}{*}{$\begin{array}{l}\text { Infraestrutura } \\
\text { de conexão da } \\
\text { Prefeitura }\end{array}$} & Computadores ligados em rede & $\begin{array}{l}0 \text { - Nenhum } \\
1 \text { - Parte } \\
2 \text { - Todos }\end{array}$ & 1,4859 & 1,6240 & 1,5813 & 1,7296 \\
\hline & Existência de intranet & $\begin{array}{l}0 \text { (Não) } \\
1 \text { (Sim) }\end{array}$ & 0,2697 & 0,3952 & 0,3155 & ,4986 \\
\hline & $\begin{array}{l}\text { Página ativa na internet do governo } \\
\text { municipal }\end{array}$ & $0-1$ & 1,0000 & 1,0000 & 1,0000 & 1,0000 \\
\hline \multirow{4}{*}{$\begin{array}{l}\text { e-Serviços e } \\
\text { Comunicação } \\
\text { com o cidadão }\end{array}$} & $\begin{array}{l}\text { Disponibiliza formas de atendimento a } \\
\text { distância }\end{array}$ & $0-1$ & ,9664 & ,9898 & ,9896 & ,9964 \\
\hline & Comunicação pela internet & $0-1$ & ,8128 & ,9230 &, 9143 & ,9613 \\
\hline & $\begin{array}{l}\text { Disponibiliza serviços informativos do } \\
\text { município e notícias }\end{array}$ & $0-1$ & ,9267 & ,9015 & ,8631 & ,9613 \\
\hline & Acesso a documentos e formulários & $0-1$ & ,1054 & ,6048 &, 5797 & ,8257 \\
\hline
\end{tabular}




\begin{tabular}{|c|c|c|c|c|c|c|}
\hline \multicolumn{7}{|c|}{ Centro final dos clusters } \\
\hline \multirow{2}{*}{ Dimensões } & \multirow{2}{*}{ Variáveis } & \multirow{2}{*}{ Range } & \multicolumn{4}{|c|}{ Cluster } \\
\hline & & & 1 & 2 & 3 & 4 \\
\hline & Licitações & $0-1$ & ,0848 & ,7826 & ,8135 & ,8709 \\
\hline & Ouvidoria serviço de atendimento ao cidadão & $0-1$ & ,1306 &, 5323 & ,3939 & ,8042 \\
\hline & Pregão eletrônico & $0-1$ & ,0138 & 2673 & 1946 & 3824, \\
\hline & Consulta a processos & $0-1$ & ,0390 & 2072 & 1890 & 4785 \\
\hline & Consulta prévia obtenção de alvará provisório & $0-1$ & ,0099 & ,0702 & ,0496 & ,2296 \\
\hline & $\begin{array}{l}\text { Diário oficial, legislação municipal e } \\
\text { finanças públicas }\end{array}$ & $0-1$ & ,1673 & ,6263 & ,6869 & ,8142 \\
\hline & Concursos públicos & $0-1$ & ,0718 & ,7067 & ,6485 & ,8379 \\
\hline & Matrícula escolar na rede pública online & $0-1$ & ,0053 & ,0091 & ,0096 & ,0387 \\
\hline & Emissão de certidão negativa de débito & $0-1$ & ,0183 &, 1416 & ,1105 & ,3565 \\
\hline & $\begin{array}{l}\text { Agendamento de consulta na rede pública } \\
\text { de saúde }\end{array}$ & $0-1$ & ,0015 &, 0057 & ,0088 & 0237 \\
\hline \multirow{7}{*}{ Inclusão digital } & $\begin{array}{l}\text { Governo desenvolve política/plano/ } \\
\text { programa inclusão digital }\end{array}$ & $0-1$ & 8113 &, 9173 & ,8118 & ,9570 \\
\hline & $\begin{array}{l}\text { Disponibiliza acesso público gratuito à } \\
\text { internet em centros de acesso }\end{array}$ & $0-1$ & ,4752 & ,6625 & ,4868 & ,7597 \\
\hline & $\begin{array}{l}\text { Infs. de serviços disponibilizados em } \\
\text { quiosque/balcão informatizado }\end{array}$ & $0-1$ & ,0168 & ,0328 & ,0192 & 0897, \\
\hline & $\begin{array}{l}\text { Estabelecer ponte entre parceiros e } \\
\text { comunidades carentes }\end{array}$ & $0-1$ & 0688, & 1042, & 0633, & 1987, \\
\hline & Criação de telecentros & $0-1$ & ,4385 & ,6127 & ,3843 & 6571 \\
\hline & $\begin{array}{l}\text { Instalação de computadores na rede } \\
\text { pública de ensino }\end{array}$ & $0-1$ & ,5264 & ,7169 & 5757, & 8307 \\
\hline & Governo garante acesso e conexão via WiFi & $0-1$ & 2460 & ,2786 & 1986 & 3659 \\
\hline \multirow{3}{*}{ e-Transparência } & $\begin{array}{l}\text { Existe legislação específica/procedimentos/ } \\
\text { providências LAl }\end{array}$ & $0-1$ & 0894, & 1789, & 1978 & ,3702 \\
\hline & $\begin{array}{l}\text { Disponibiliza meios para solicitação/acesso } \\
\text { a informação pública }\end{array}$ & $0-1$ & ,7838 & 8913 & ,9127 & ,9720 \\
\hline & $\begin{array}{l}\text { Solicitações recebidas governo municipal } \\
\text { (2013) }\end{array}$ & $0-1$ & 1795 & ,3035 & 2282 & 4770 \\
\hline
\end{tabular}




\begin{tabular}{|c|c|c|c|c|c|c|}
\hline \multicolumn{7}{|c|}{ Centro final dos clusters } \\
\hline \multirow{2}{*}{ Dimensões } & \multirow{2}{*}{ Variáveis } & \multirow{2}{*}{ Range } & \multicolumn{4}{|c|}{ Cluster } \\
\hline & & & 1 & 2 & 3 & 4 \\
\hline & $\begin{array}{l}\text { Disponibiliza em tempo real informações } \\
\text { da execução orçamentária e financeira }\end{array}$ & $0-1$ & ,6066 & ,6784 &, 9143 & ,9397 \\
\hline & Registro das competências & $0-1$ & ,2498 & ,2446 & ,3042 & ,7432 \\
\hline & Estrutura organizacional & $0-1$ &, 4775 & ,4609 &, 5044 & 8788 \\
\hline & $\begin{array}{l}\text { Endereços e telefones das unidades } \\
\text { organizacionais }\end{array}$ & $0-1$ & ,6211 & ,6988 & ,7590 & 9591 \\
\hline & Horários de atendimento ao público & $0-1$ & ,3293 & ,3431 & ,3731 & 8164 \\
\hline & $\begin{array}{l}\text { Registros de repasses ou transferências de } \\
\text { recursos financeiros }\end{array}$ & $0-1$ & ,3461 & 0566 & 8447 & 8307 \\
\hline & Registros das despesas & $0-1$ & ,4332 & , 1846 &, 9544 & ,9283 \\
\hline & $\begin{array}{l}\text { Informações concernentes a procedimentos } \\
\text { licitatórios }\end{array}$ & $0-1$ & ,3407 & ,4519 & , 8038 & ,8759 \\
\hline & $\begin{array}{l}\text { Dados gerais para o acompanhamento de } \\
\text { programas }\end{array}$ & $0-1$ & ,2857 & 1971 & ,3747 & , 6600 \\
\hline & $\begin{array}{l}\text { Respostas às perguntas mais frequentes } \\
\text { da sociedade }\end{array}$ & $0-1$ & 1268 & ,1166 & ,1105 & 4204 \\
\hline & Publicados em formato aberto & $0-1$ & ,4607 &, 5640 &, 5669 & 6686 \\
\hline & Há atualização de dados & $0-1$ & ,7785 & ,7497 & , 8695 & ,8852 \\
\hline \multirow{7}{*}{$\begin{array}{l}\text { Caracterização } \\
\text { dos municípios }\end{array}$} & Número de municípios por cluster & & 1309 & 883 & 1249 & 1394 \\
\hline & Média da população (2014) & & 17.490 & 28.960 & 22.079 & 83.190 \\
\hline & Média do PIB per capita (2012) & & $11.148,28$ & $15.178,27$ & $15.635,41$ & $19.118,47$ \\
\hline & Média de IDH-M (2010) & & 0,634 & 0,668 & 0,670 & 0,698 \\
\hline & Média de Receitas per capita (2014) & & $2.834,80$ & $3.144,16$ & $3.314,22$ & $3.174,17$ \\
\hline & & Legenda & \multicolumn{4}{|c|}{ Piores resultados } \\
\hline & & & & Melhores : & sultados & \\
\hline
\end{tabular}

Fonte: Elaborada pelos autores.

Os resultados permitem observar os municípios em agrupamentos que foram denominados: o Sem-tecnologia, o Atento ao cidadão, o Atento à legislação e o Provido de TIC. Além desses, há um quinto conjunto composto pelos 735 municípios excluídos da amostra. 
1) Cluster 1 - o Sem-tecnologia

O Sem-tecnologia tem média populacional de 17.490 habitantes, um PIB per capita médio de R\$ $11.148,00$, com receita per capita média, por município, de $\mathrm{R} \$ 2.834,00$ e a pior média do IDH-M: 0,634. É o menor e o mais pobre. Apresenta os piores indicadores de uso e infraestrutura de TIC: a prefeitura possui pouca infraestrutura, os serviços eletrônicos e a comunicação com a população têm baixos índices, promove pequeno número de ações de inclusão digital e de e-transparência. O grupo é formado por 1.309 municípios e, embora o maior número esteja na região Nordeste (514), eles existem em todas as regiões brasileiras.

2) Cluster 2 - o Atento ao cidadão

O grupo Atento ao cidadão (883 municípios) tem média populacional de 28.960 habitantes, com PIB per capita de R $\$ 15.178,00$, receitas per capita do município em torno de R $\$ 3.144,00$ e IDH -M 0,668. Mantém esforços em direção ao cidadão, pois utiliza a TIC para promover e-serviços e comunicação. Dá alguma atenção à inclusão digital e pouca à transparência por meios eletrônicos. Grande parte dos municípios do grupo Atento ao cidadão está na região Sudeste (297).

3) Cluster 3 - o Atento à legislação

Possui PIB per capita médio de R\$15.635,00 e 22.079 habitantes, em média; receita per capita municipal média de $\mathrm{R} \$ 3.314,00$, a melhor entre os quatro grupos, e IDH-M de 0,670. Esse município é maior e mais rico do que os anteriores. Por ter melhores condições econômicas, pode avançar na infraestrutura de conexão da prefeitura, na oferta de e-serviços aos cidadãos, na comunicação e na inclusão digital. Está mais preocupado com transparência do que com o atendimento ao cidadão, possivelmente para cumprir a legislação. Grande número dos municípios desse cluster também é da região Sudeste (408). O grupo é formado por 1.249 municípios e está em segundo lugar nos indicadores socioeconômicos.

4) Cluster 4 - o Provido de TIC

O Provido de TIC representa o grupo de 1.394 municípios que apresentou o melhor resultado, em relação aos indicadores socioeconômicos, com PIB per capita médio de R $\$ 19.188,00$, receitas per capita municipais de R\$3.174,00, inferior ao cluster 3, e IDH-M 0,698. Concentra capitais e cidades importantes no contexto nacional, como São Paulo e Rio de Janeiro. Esses dois municípios tiveram os indicadores socioeconômicos (PIB, população e receitas) retirados do cálculo das médias a fim de evitar distorção. O Provido de TIC é um município grande e rico, média de 83.190 habitantes, todos os seus indicadores de TIC são os melhores, da infraestrutura ao uso para o cidadão e à e-transparência. O grupo é formado em grande parte por municípios do Sul (471) e do Sudeste (415).

5) Os excluídos da amostra

Os 735 municípios excluídos da análise são pequenos, média de 14.706 habitantes, e apresentam piores indicadores socioeconômicos que os dos grupos anteriores. O PIB per capita médio é de R\$ 9.298,00, as receitas per capita municipais médias são de R\$2.628,00, IDH-M é de 0,597. Desses municípios, $85,3 \%$ dizem que a página do governo municipal não está em funcionamento. Sobre a inclusão digital, 77,2\% afirmaram desenvolver alguma ação, embora de forma tímida, 44,9\% possuem telecentros, $49,7 \%$ possuem computador na rede pública de ensino, 17,9\% disponibilizam acesso a conexão Wi-Fi. Sobre a transparência, 95\% ainda não possuem legislação específica para atendimento à LAI. 
Os municípios brasileiros têm características distintas de uso de TIC, o que enseja a discussão de diferentes problemáticas e possibilidades de ação.

\section{DISCUSSÃO DOS RESULTADOS}

Há limitações no uso de TIC pelos municípios, mas houve avanços em relação ao cenário de 2009 e 2012 (Sano, 2012; Przeybilovicz, Cunha e Quandt, 2014; Przeybilovicz, Silva e Cunha, 2015). A obrigatoriedade de interação eletrônica exigida pelo governo federal aos municípios, em alguns programas, pode ter universalizado o uso de computador e internet, o que provocou inclusive a retirada dessas variáveis do modelo de análise, mas o uso dos recursos é distinto. O limitado uso de TIC por grande parte dos municípios pode conduzir a investimentos em iniciativas pouco eficientes de eGov e smart cities. Há mais de uma década, numa reflexão sobre a pesquisa de eGov, Grönlund (2005) mostrou que a disponibilidade de computador e acesso à internet por governos não fez com que os cidadãos tivessem melhores serviços e/ou os governos ganhassem melhor gestão dos recursos. Não necessariamente, ainda que em alguns casos isso tenha acontecido. Na discussão dos resultados deste trabalho, propõem-se diferentes abordagens de fomento ao uso de TIC para o Sem-tecnologia, o Atento ao cidadão, o Atento à legislação e o Provido de TIC.

Uma política de eGov com transparência, e-serviços e participação exige recursos mínimos de infraestrutura de TIC, que o Sem-tecnologia não possui. Nos países em desenvolvimento ou emergentes, assim como no Brasil, o uso de TIC é um problema especialmente espinhoso. Municípios pobres e pequenos dependem fortemente de recursos de outros níveis de governo e, por conta própria, dificilmente conseguem desenhar políticas públicas que se beneficiem de tecnologia. Levando em consideração a oportunidade de criação interativa, participativa e apoiada por TIC (Batagan, 2011), a falta de infraestrutura, gestão e habilidades em TIC os deixa pouco habilitados a atingir o status de uma smart city (Batty et al., 2012). Os resultados deste estudo, e uma comparação com estudos internacionais, permitem algumas sugestões de governança de TI para o município Sem-tecnologia. Várias barreiras para o desenvolvimento de iniciativas de eGov foram relatadas na literatura, incluindo a falta de recursos financeiros, recursos técnicos, pessoal qualificado e tamanho do município (Moon, 2002). Embora não seja justo atribuir a culpa da falta de informatização ao governo central, ele tem um papel estratégico sobre algumas destas questões (Rahman, 2010). Por outro lado, em vez de depender exclusivamente do Estado ou financiamento federal, o município Sem-tecnologia pode formar parcerias com outros municípios da região, consórcios, ou ainda formar grupos de municípios e buscar financiamento de agências internacionais (Thompson e Brown, 2007) para ampliar a disponibilidade e uso de TIC, em eGov ou smart cities, e para incentivar a inclusão digital. O governo central pode fornecer suporte a essas iniciativas, agindo como garantidor de empréstimos e oferecendo apoio técnico à elaboração de projetos locais. Com o estabelecimento de um sistema nacional, temporário, é possível apoiar o desenvolvimento dessas iniciativas, com pessoas qualificadas na utilização das TIC e na formulação de políticas públicas para o desenvolvimento de sistemas de informação e apoio à implementação. O município Sem-tecnologia é o alvo, ele necessita de maior atenção das associações de municípios e dos governos estadual e federal, para promover políticas públicas e implementar recursos de TIC. Oferecer catálogos de soluções para municípios, incentivar o uso de soluções já disponíveis na forma de software livre, promover formação e apoio ao uso, sugerir implantação de práticas e processos de gestão de TI, em ciclos curtos, para obtenção de ganhos rápidos, apoio finan- 
ceiro e para investimento, e uma sugestão de marco legal. Ter a disponibilidade da TIC não garante o desenvolvimento de eGov (Gil-Garcia, Arellano-Gault e Luna-Reyes, 2010; Rahman, 2010) e smart cities (Nam e Pardo, 2011), mas sem ela há pouca esperança de alcançar os benefícios da oferta de e-serviços, de e-democracia (Turner, 2005) e de transformar a vida e o trabalho na região de forma significativa (Komninos e Sefertzi, 2009). O Sem-tecnologia requer ampliação do uso de tecnologia e desenvolvimento de capacidades institucionais em ciclos curtos e com resultados percebidos pela gestão e/ou população. A autoaprendizagem tem um ciclo longo demais para esse município, os riscos de investimento são altos, e é preciso considerar a falta de mobilização local nesta direção. É importante ressaltar que a forma de implantação das ações de gestão de TI, participativa e a partir das necessidades locais, pode atenuar os efeitos da criação de dependência técnica e de recursos do governo central e obter o comprometimento da população.

O Atento ao cidadão é um grupo de municípios que "faz a lição de casa" e que, na concorrência entre escassez de recursos e demandas municipais, aplica os recursos de TIC em direção ao cidadão. É possível imaginá-lo com melhor gestão de TIC que o grupo anterior e já com capacidade institucional e com alguns processos de gestão de TI. É de se esperar que a população aproveite melhor os benefícios potenciais das ações de eGov, pois há maior oferta de e-serviços e mais iniciativas de inclusão digital. O município precisa avançar em e-transparência, uma dimensão importante para exercício de controle social sobre as ações do governo local. O Atento ao cidadão é um município com potencial para iniciativas smart.

Os resultados também mostram que não é suficiente ter recursos e infraestrutura de TIC para aumentar as ações de eGov, como é o caso do Atento à legislação. Esse cluster é composto de municípios com relativas boas condições socioeconômicas, com alguns municípios entre aqueles de maior receita. Precisa incluir em sua agenda ações de TIC voltadas ao cidadão, especialmente prestação de e-serviços e inclusão digital. O Brasil já tem política de transparência pública com leis federais, sendo a Lei de Acesso à Informação (LAI, Lei $n^{\circ}$ 12.527) a mais recente, promulgada em novembro de 2011. Os governos federal, estaduais e municipais estão sujeitos à LAI, e ela foi implementada em estágios. Tornou-se obrigatória para os grandes municípios, quando entrou em vigor (maio de 2012) e, progressivamente, a obrigatoriedade estendeu-se a todos os demais (2014). A obrigação legal pode ser uma explicação para o Atento à legislação ter avançado em e-transparência, não priorizando o uso de TIC no atendimento ao cidadão.

Os clusters dois e três, o Atento ao cidadão e o Atento à legislação, possuem condições para oferecer melhores serviços eletrônicos aos cidadãos. O estabelecimento de uma plataforma horizontal de gestão dos recursos de TIC melhora o compartilhamento de informações, para prestar serviços públicos, de forma mais eficiente e transparente. Aumenta as economias de escala e a capacidade de negociação com fornecedores. No entanto, pode não haver demanda, pelos cidadãos, por esses serviços e também não há um marco legal exigindo que os municípios os ofereçam. Assim, a prestação de serviços eletrônicos é diferente entre os dois grupos. Há que se pensar em padrões de atendimento aos cidadãos pelos meios eletrônicos e de governança dos recursos de TI, que no início contemplem incentivos à adoção, mas que progridam rumo à obrigatoriedade/regulação e universalização de serviços, em um prazo factível.

O Provido de TIC é o de maior potencial para ser uma smart city. É o município com os melhores indicadores de recursos e uso de TIC. O banco de dados utilizado não permite uma avaliação de todas as dimensões do modelo conceitual de eGov (Cunha e Miranda, 2013), mas o grupo teve 
bons resultados nas quatro dimensões analisadas. Nesse cluster estão algumas capitais e municípios importantes no contexto nacional e que já possuem iniciativas smart. Uma vez que a cidade tenha uma infraestrutura de tecnologia e prestação de serviços ao cidadão, poderá interconectar cidadãos, serviços urbanos e os outros atores da cidade, como empresas, terceiro setor, universidades, coletivos. Capturam-se sinergias entre os serviços públicos já prestados e também a melhoria e/ou desenvolvimento de novos serviços para o cidadão e para as empresas. Do cruzamento e análise dos diferentes dados da cidade, serviços e cidadãos, facilita-se a tomada de decisão e aperfeiçoa-se a gestão da cidade. Ao fornecer informações em tempo real aos cidadãos, estes podem tomar suas decisões pessoais com base no que está acontecendo ao redor. Exemplos de serviços desse tipo são os de mobilidade, como aplicativos de ônibus ou de trânsito. Os municípios desse cluster possuem infraestrutura para desenvolver uma arquitetura para uma plataforma tecnológica robusta, padrão, aberta e interoperável que faça com que o município não se torne refém de fornecedores e assegure o desenvolvimento de um ecossistema inovador na smart city. Esses municípios são os que se mostram minimamente habilitados a desenvolver novas formas de contratação de fornecedores, como parcerias público-privadas (PPPs) e gestão de contratos (ou outras formas de acordos) com estabelecimento de níveis de serviços tecnológicos.

Por fim, há os 735 municípios sem cluster, possivelmente excluídos digitais, com os piores indicadores socioeconômicos e indícios de precária infraestrutura e uso de TIC. Eles possuem desafios ainda maiores para desenvolver suas ações de eGov e smart city. É importante para os municípios mais pobres, como o Sem-tecnologia e o excluído digital, receber ajuda para que possam desfrutar dos benefícios de melhoria crescente na gestão pública e de seus indicadores sociais.

\section{CONSIDERAÇÕES FINAIS}

Este estudo orientou-se pela indagação de como a infraestrutura e o uso de TIC caracterizam grupos de municípios. Os resultados mostraram que existem quatro clusters e suas características, com consequências para as iniciativas de eGov e smart cities. O estudo analisou o perfil dos municípios a partir de quatro dimensões (Cunha e Miranda, 2013): infraestrutura de conexão da prefeitura, eserviços e comunicação com o cidadão, inclusão digital e e-transparência.

O trabalho buscou contribuir teoricamente, descrevendo uma tipologia de municípios a partir das características de infraestrutura e uso de TIC. Os resultados mostram quatro tipos com diferentes necessidades. Enquanto o Sem-tecnologia requer condições básicas de TIC, o Atento à legislação precisa melhorar a forma como as TIC podem ser gerenciadas e direcionadas para facilitar a vida do cidadão. O Atento ao cidadão precisa ampliar as ações de e-transparência. O Provido de TIC encontra-se em nível mais avançado e pode continuar as ações voltadas para a perspectiva de smart cities.

A pesquisa em eGov deve ter reflexo na prática da governança de TI nos governos municipais, e ainda mais nos países em desenvolvimento (Grönlund, 2005). Segundo o governo federal, ${ }^{1}$ as iniciativas de TIC nos governos visam a democratização do acesso à informação e devem seguir três diretrizes fundamentais: a participação dos cidadãos, a melhoria da gestão interna e a integração com parceiros e fornecedores. No entanto, recomendações genéricas não servem a todos os municípios. As políticas

\footnotetext{
${ }^{1}$ Disponível em: <www.governoeletronico.gov.br/>.
} 
públicas de eGov recomendando princípios genéricos, no Brasil, podem não ter surtido os efeitos que se esperava e o mesmo pode vir a acontecer com as iniciativas de smart city. A investigação de eGov e smart cities, em países em desenvolvimento, deve considerar os degraus que municípios precisam galgar. Os desafios não são triviais e as ações que considerem as diferenças entre eles podem alavancar os resultados. Os governos municipais são responsáveis pela definição de políticas públicas, segundo as prioridades locais. O uso de TIC pode mudar o potencial econômico da região e criar maior competitividade, levando a novas oportunidades de negócios e inovação, influenciando o potencial econômico de um município e a possibilidade de que ele venha a se tornar uma smart city. Os esforços devem ser conscientes para usar a TIC com o objetivo de transformar a vida e o trabalho em uma região de forma significativa. É importante para os municípios mais pobres receberem das outras esferas de governo ajuda financeira e/ou recursos para investimento em infraestrutura básica de TIC.

Foram identificados diferentes grupos de municípios, cada um com requisitos específicos para a melhor utilização das TIC. Uma das limitações do estudo é o fato de as variáveis que compõem cada dimensão terem sido extraídas de uma fonte secundária, a Munic 2014, que não define completamente o uso das TIC nos municípios. A Munic 2014 teve alteração no conjunto de questões feitas aos municípios, o que impossibilitou a comparação aprofundada com estudos anteriores e diminuiu a capacidade de discriminação entre os grupos de municípios. Mesmo assim, a Munic é um estudo nacional que tem sido conduzido regularmente por certo número de anos e, por conseguinte, permite alguma comparação. Parece ter ocorrido uma evolução no uso de TIC pelos municípios, entre 2009 (Sano, 2012), 2012 e 2014 (Przeybilovicz, Silva e Cunha, 2015). Esta é uma das sugestões para estudos futuros: analisar como a utilização das TIC nos municípios evolui, usando a mesma base de dados, comparando estados, regiões e municípios, e efetuar análises dentro dos agrupamentos encontrados neste trabalho. Outros estudos podem buscar analisar mais profundamente a aproximação dos conceitos e características de desenvolvimento e implantação de eGov e projetos de smart city. Olhar a literatura de eGov pode lançar luz sobre como o termo e as iniciativas de smart city estão sendo apropriados no contexto brasileiro. Perspectivas de outros campos de conhecimento, como ciência política, gestão pública, gestão urbana e sociologia, podem complementar a pesquisa sobre governo eletrônico e smart cities. 


\section{REFERÊNCIAS}

ALMAZAN, Rodrigo S.; CASTAÑED, Miguel A. Gobierno electrónico en los municipios: el caso de Toluca. In: ANNUAL INTERNATIONAL DIGITAL GOVERNMENT RESEARCH CONFERENCE ON PUBLIC ADMINISTRATION, 11., 2010, México. Proceedings... México: Digital Government Society of North America, 2010. p. 245-246.

ANTTIROIKO, Ari-Veikko. Urban planning 2.0. International Journal of E-Planning Research (IJEPR), v. 1, n. 1, p. 16-30, 2012.

ASOGWA, Brendan E. Electronic government as a paradigm shift for efficient public services: opportunities and challenges for Nigerian government. Library Hi Tech, v. 31, n. 1, p. 141-159, 2013.

BATAGAN, Lorena. Smart cities and sustainability models. Informatica Economica, v. 15, n. 3, p. 80-87, 2011.

BATTY, Michael et al. Smart cities of the future. The European Physical Journal Special Topics, v. 214, n. 1, p. 481-518, 2012.

BERNARDES, Maciele B.; SANTOS, Paloma M.; ROVER, Aires J. Ranking das prefeituras da região Sul do Brasil: uma avaliação a partir de critérios estabelecidos na Lei de Acesso à Informação. Rev. Adm. Pública, Rio de Janeiro, v. 49, n. 3, p. 761-792, 2015.

BOLÍVAR, Manuel P. R. Characterizing the role of governments in smart cities: a literature review. In: GIL-GARCIA, Juan R.; PARDO, Theresa A.; NAM, Taewoo (Ed.). Smarter as the new urban agenda: a comprehensive view of the 21 st century city. Nova York: Springer, 2016.

CASTELLS, Manuel; HALL, Peter. Technopoles of the world. Londres: Routledge, 1994.

CGI. Comitê Gestor da Internet no Brasil. Pesquisa sobre o uso de tecnologias de informação e comunicação. TIC governo eletrônico 2015. São Paulo: Comitê Gestor da Internet no Brasil, 2016a.

CGI. Comitê Gestor da Internet no Brasil. Pesquisa sobre o uso de tecnologias de informação e comunicação no Brasil. TIC domicílios e empresas 2015. São Paulo: Comitê Gestor da Internet no Brasil, 2016b.

CHOURABI, Hafedh et al. Understanding smart cities: an integrative framework. In: HAWAII
INTERNATIONAL CONFERENCE ON SYSTEM SCIENCE, 45., 2012, Havaí. Proceedings... Havaí: HICSS, 2012. p. 2289-2297.

COMISSÃO EUROPEIA. European Innovation Partnership on Smart Cities and Communities: Strategic Implementation Plan. Smart City and Communities, v. 1, n. 1, p. 1-22, 2012.

CUNHA, Maria A.; MIRANDA, Paulo R. de M. A pesquisa no uso e implicações sociais das tecnologias da informação e comunicação pelos governos no Brasil: uma proposta de Agenda a partir de reflexões da prática e da produção acadêmica nacional. O\&S - Organizações \& Sociedade, v. 66, n. 20, p. 543-566, jul./set. 2013.

CUNHA, Maria A. et al. Smart cities: transformação digital de cidades. São Paulo: Programa Gestão Pública e Cidadania, 2016.

DAMIAN, Ieda P. M.; MERLO, Edgard M. Uma análise dos sites de governos eletrônicos no Brasil sob a ótica dos usuários dos serviços e sua satisfação. Rev. Adm. Pública, Rio de Janeiro, v. 47, n. 4, p. 877-900, 2013.

DINIZ, Eduardo H. et al. O governo eletrônico no Brasil: perspectiva histórica a partir de um modelo estruturado de análise. Rev. Adm. Pública, Rio de Janeiro, v. 43, n. 1, p. 23-48, 2009.

GIFFINGER, Rudolf; GUDRUN, Haindlmaier. Smart cities ranking: an effective instrument for the positioning of the cities? ACE: Architecture, City and Environment, v. 4, n. 12, p. 7-26, 2010.

GIL-GARCIA, Juan R.; ARELLANO-GAULT, David; LUNA-REYES, Luis F. Gobierno electrónico en México (2000-2006): una visión desde la nueva gestión pública. In: ANNUAL INTERNATIONAL DIGITAL GOVERNMENT RESEARCH CONFERENCE ON PUBLIC ADMINISTRATION, 11., 2010, México. Proceedings... México: Digital Government Society of North America, 2010. p. 163-172.

GONÇALVES, Flávio S. Infraestrutura de acesso à internet: o arranjo político-econômico do NavegaPará. 2011. 218 f. Dissertação (mestrado em comunicação) - Programa de Pós-Graduação em Comunicação, Faculdade de Comunicação, Universidade de Brasília, Brasília, 2011.

GRÖNLUND, Åke. State of the art in e-Gov research: surveying conference publications. International 
Journal of Electronic Government Research, v. 1, n. 4, p. 1-25, 2005.

GRÖNLUND, Åke. Ten years of e-government: the 'end of history' and new beginning. In: INTERNATIONAL CONFERENCE ON ELECTRONIC GOVERNMENT, 9., 2010, Lausanne. Proceedings... Lausanne: Springer, 2010. p. 13-24.

GREGOR, Shirley. The nature of theory in information systems. MIS Quarterly, v. 30, n. 3, p. 611-642, 2006.

HAIR, Joseph F. et al. Multivariate data analysis. 5. ed. Nova Jersey: Prentice-Hall, 1998.

HOLLANDS, Robert G. Will the real smart city please stand up? City, v. 12, n. 3, p. 303-320, 2008.

IBGE. Instituto Brasileiro de Geografia e Estatística. Pesquisa nacional de amostra por domicílio. Disponível em: <www.ibge.gov.br/home/estatistica/ economia/perfilmunic/2015>. Acesso em: 4 out. 2016.

JAMBEIRO, Othon; SOBREIRA, Roseane; RABEIRO, Priscila. TICs e gestão pública em planos diretores de cidades brasileiras. Revista Eptic Online, v. 11, n. 3, 2011.

KAYLOR, Charles; DESHAZO, Randy; VAN ECK, David. Gauging e-government: a report on implementing services among American cities. Government Information Quarterly, v. 18, n. 4, p. 293-307, 2002.

KOMNINOS, Nicos; SEFERTZI, Elia. Intelligent cities: R\&D offshoring, Web 2.0 product development and globalization of innovation systems. In: KNOWLEDGE CITIES SUMMIT, 2., 2009, Shenzhen. Proceedings... Shenzhen: MAKCi, 2009. p. 1-8.

LALOVIĆ, Ksenija; DJUKANOVIĆ, Zoran; ZIVKOVIC, Jelena. Building the ICT fundament for local E-government in Serbia-Municipality of Loznica example. In: CORP 2004: GEO MULTIMEDIA 04, 2004, Viena. Proceedings... Viena: Real Corp, 2004.

LEENES, Ronald; SVENSSON, Jorgen. Size matterselectronic service delivery by municipalities? In: INTERNATIONAL CONFERENCE ON ELECTRONIC GOVERNMENT, 2002, France. Proceedings... France: Springer, 2002. p. 150-156.
MALEK, Jalalauddin A. Informative global community development index of informative smart city. In: INTERNATIONAL CONFERENCE ON EDUCATION AND EDUCATIONAL TECHNOLOGY, 8., 2009, Italia. Proceedings... Gênova: WSEAS, 2009. p. 17-19.

MCFARLANE, C. Learning the city: knowledge and translocal assemblage. Oxford: Wiley-Blackwell, 2011.

MEIJER, Albert; BOLÍVAR, Manuel P. R. Governing the smart city: a review of the literature on smart urban governance. International Review of Administrative Sciences, v. 82, n. 2, p. 392-408, 2016.

MEIRELLES, Fernando S. Administração e uso de TI: tecnologia da informação nas empresas. 27. ed. São Paulo: Fundação Getulio Vargas, 2016.

MOON, Michel J. The evolution of e-government among municipalities: rhetoric or reality? Public Administration Review, v. 62, n. 4, p. 424-433, 2002.

NAM, Taewoo; PARDO, Theresa A. Conceptualizing smart city with dimensions of technology, people, and institutions. In: ANNUAL INTERNATIONAL DIGITAL GOVERNMENT RESEARCH CONFERENCE, 12., 2011, Nova York. Proceedings... Nova York: ACM, 2011.p. 282-291.

NGULUBE, Patrick. The nature and accessibility of e-government in sub-Saharan Africa. International Review of Information Ethics, v. 7, n. 9, p. 1-13, 2007.

PINHO, José A. G. de. Investigando portais de governo eletrônico de estados no Brasil: muita tecnologia, pouca democracia. Rev. Adm. Pública, Rio de Janeiro, v. 42, n. 3, p. 471-493, 2008.

PRZEYBILOVICZ, Erico; CUNHA, Maria A.; QUANDT, Carlos O. Perfil dos municípios brasileiros em relação ao uso e à infraestrutura de TIC: uma análise dos clusters. In: ENCONTRO DA ANPAD, 38., 2014, Rio de Janeiro. Anais... Rio de Janeiro: Anpad, 2014.

PRZEYBILOVICZ, Erico; SILVA, Wesley V. da; CUNHA, Maria A. Limits and potential for egov and smart city in local government: a cluster analysis concerning ICT infrastructure and use. International Journal of E-Planning Research (IJEPR), v. 4, n. 2, p. 39-56, 2015.

RAHMAN, Hakikur. Framework of e-governance at the local government level. In: REDDICK, 
Christopher G. (Ed.). Comparative e-government. Nova York: Springer, 2010. p. 23-47.

RAMPELOTTO, Alexandre; LÖBLER, Mauri L.; VISENTINI, Monize S. Avaliação do sítio da Receita Federal do Brasil como medida da efetividade do governo eletrônico para o cidadão. Rev. Adm. Pública, Rio de Janeiro, v. 49, n. 4, p. 959-983, jul./ago. 2015.

SANO, Hironobu. Governo eletrônico nos municípios brasileiros: um conto de fadas da web 1.0? In: ENCONTRO NACIONAL DE ADMINISTRAÇÃO PÚBLICA E GOVERNO, 5., 2012, Salvador. Anais... Salvador: ENAPG, 2012. p. 1-16.

SIAU, Keng; LONG, Yuan. Factors impacting e-government development. Journal of Computer Information Systems, v. 50, n. 1, p. 98-107, 2009.

SINGH, Harminder; DAS, Aamit; JOSEPH, Damien. Country-level determinants of e-government maturity. Communications of the Association for Information Systems, v. 20, n. 1, p. 632-648, 2007.

SRIVASTAVA, Shirish C.; TEO, Thompson S. H. E-government, e-business, and national economic performance. Communications of the Association for Information Systems, v. 26, n. 1, p. 267-286, 2010.
THOMPSON, Sheryl; BROWN, David. Jamaica: implementing the national ICT strategy through policy and initiatives. In: AMERICAS CONFERENCE ON INFORMATION SYSTEMS, 2007, Colorado. Proceedings... Colorado: AMCIS, 2007. p. 1-9.

TRIDAPALLI, Juarez P.; FERNANDES, Elton; MACHADO, Waltair V. Gestão da cadeia de suprimento do setor público: uma alternativa para controle de gastos correntes no Brasil. Rev. Adm. Pública, Rio de Janeiro, v. 45, n. 2, p. 401-33, mar./ abr. 2011.

TURNER, Timothy J. Local government e-disclosure \& comparisons: equipping deliberative democracy for the 21st century. Nova York: University Press of America, 2005.

UNITED NATIONS. Department of Economic. United Nations e-governmet survey 2016: e-government in support of sustainable development. Nova York: United Nations Publications, 2016.

ZHAO, Fang; COLlIER, Alan; DENG, Hepu. A multidimensional and integrative approach to study global digital divide and e-government development. Information Technology \& People, v. 27, n. 1, p. 3862,2014

\section{Erico Przeybilovicz}

Doutorando em administração de empresas na Escola de Administração de Empresas de São Paulo da Fundação Getulio Vargas. E-mail: erico.prz@fgv.br.

\section{Maria Alexandra Cunha}

Doutora em administração de empresas e professora na Escola de Administração de Empresas de São Paulo da Fundação Getulio Vargas. E-mail: alexandra.cunha@fgv.br.

\section{Fernando de Souza Meirelles}

Doutor em administração e professor titular na Escola de Administração de Empresas de São Paulo da Fundação Getulio Vargas. E-mail: fernando.meirelles@fgv.br. 